\title{
Opisthotonus (arc de cercle) in anti-NMDAR encephalitis
}

\section{Opistótono (arc de cercle) em um caso de encefalite anti-NMDAR}

Guillermo Delgado-García ${ }^{1}$, Vanessa Cano-Nigenda ${ }^{1}$, Arturo Abundes-Corona ${ }^{1}$, Karina Carrillo-Loza ${ }^{1,2}$, Juan Calleja-Castillo', José Flores-Rivera ${ }^{1,2}$

A 21-year-old man presented with focal-onset seizures with evolution to bilateral tonic-clonic seizures, fever, and encephalopathy. In our hospital, he was diagnosed with anti-NMDAR encephalitis; this antibody was found to be positive in both serum and cerebrospinal fluid (CBA, IIFT, EUROIMMUN AG, Luebeck, Germany). Other causes of encephalitis were rationally excluded. During his hospital stay, he developed symptoms and signs compatible with catatonia. He was treated with high-dose methylprednisolone pulse therapy and therapeutic plasma exchange. Although he comparatively improved, days after finishing his fifth session of therapeutic plasma exchange, he developed a brief episode of opisthotonus (arc de cercle) (Figure), which resolved without any treatment. He had never had similar episodes in the past. This movement disorder is infrequently seen in contemporary clinical practice. In the 19th century, hysteria was one of the major differential diagnoses of episodic opisthotonus. ${ }^{1}$

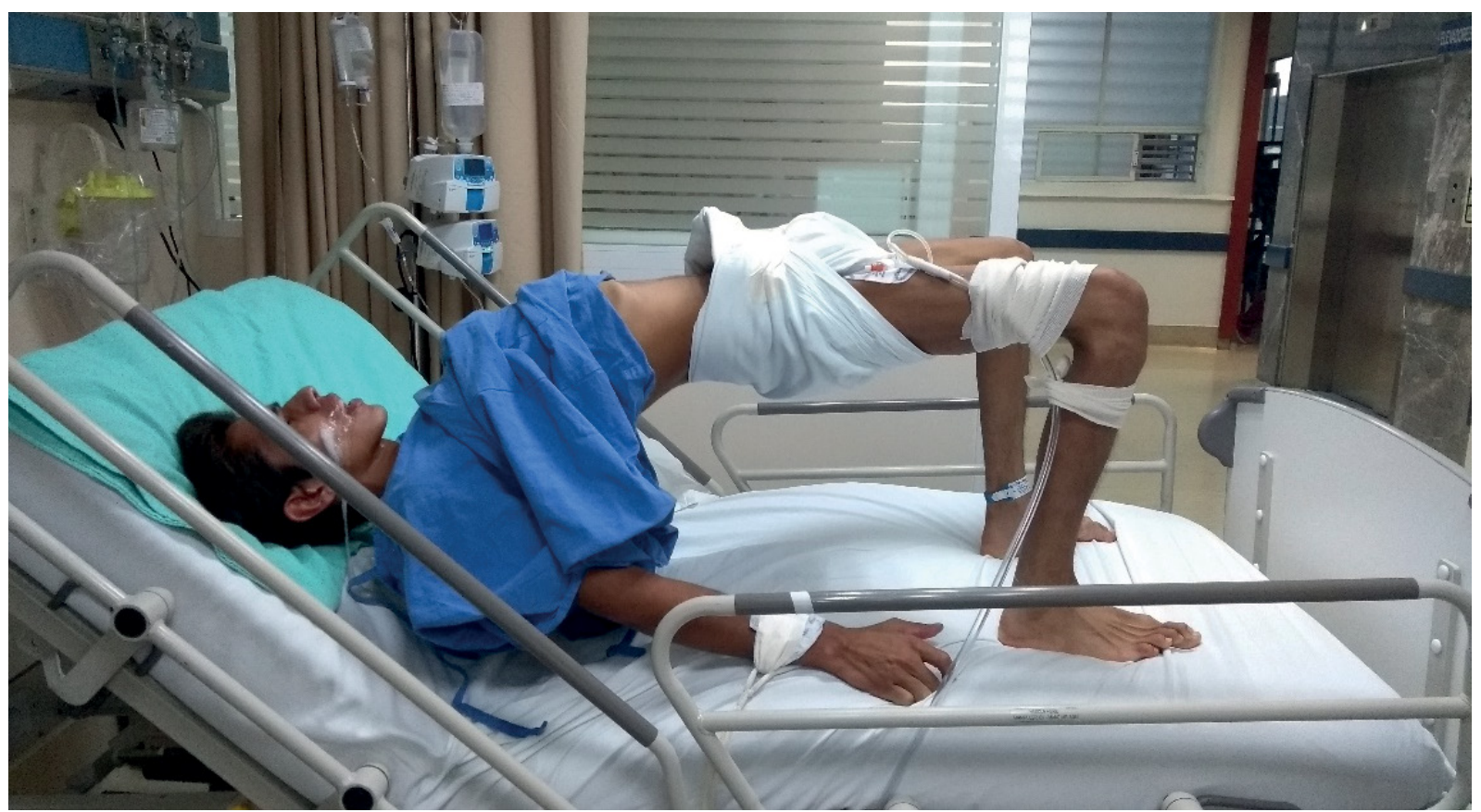

Figure. Episodic opisthotonus (arc de cercle) in anti-NMDAR encephalitis. The patient presented with a severe hyperextension of the neck and back, which led him to an "arching" position. This episode lasted a few seconds and resolved without treatment. 